\title{
EL REPARTO DE LOS TRABAJOS DOMÉSTICOS Y DE CUIDADOS COMO TERMÓMETRO DE LA PARIDAD EN LAS RELACIONES DE PAREJA
}

\author{
AMAiA AgIRRE MiguÉLEZ \\ Universidad del País Vasco/Euskal Herriko Unibertsitatea
}

Recibido: 24/03/2014

Aceptado: 12/05/2014

\section{Resumen}

Este artículo presenta algunos de los resultados de una investigación cualitativa sobre las negociaciones en relaciones de pareja paritarias. Más concretamente, se centra en las negociaciones acerca del trabajo doméstico y de cuidados, y de cómo el reparto de este trabajo sirve de termómetro para medir el nivel de igualdad de la relación. Según las personas entrevistadas, no se negocia este reparto del trabajo de manera explícita, y tienden a definirlo como un reparto «natural» y no premeditado, ya que su explicitación choca frontalmente con el ideal de amor romántico imperante en nuestra sociedad. Aún así, las mujeres entrevistadas plantean ansias de cambio y conflictos al respecto, ya que el no negociar abiertamente redunda en un reparto desigual basado en los roles tradicionales de género e impide avanzar en la igualdad entre mujeres y hombres.

Palabras clave: Feminismo, parejas, negociación, trabajo doméstico, trabajo de cuidados, conflictos.

\begin{abstract}
This article presents some of the results of a larger qualitative piece of research about negotiations in egalitarian couples. More specifically, it focuses on the negotiations about the housework and care work, since its distribution works as a thermometer for gender equality. According to the respondents, work distribution is not explicitly negotiated; it is rather defined as a 'natural', non-premeditated distribution, because this explicitness would confront the ideal of romantic love in our society. Yet, the female interviewees express a wish for change and reveal an underlying conflict: the lack of
\end{abstract}


open negotiation leads to an unequal distribution based on traditional gender roles, thus impeding progress on gender equality.

Keywords: feminism, couples, negotiation, housework, care work, conflicts.

Feminismo/s 23, junio 2014, pp. 91-110 


\section{Introducción}

Este artículo forma parte de una investigación más amplia sobre los procesos de negociación de parejas con ideología paritaria en el País Vasco ${ }^{1}$. Concretamente se han realizado entrevistas en profundidad a 15 parejas (30 personas, ya que las entrevistas se han realizado por separado) con el objetivo de conocer si existen nuevos modelos emergentes de relaciones de pareja y comprobar cuáles son las relaciones negociadoras que se llevan a cabo. Nos parece fundamental acercarnos a estos procesos negociadores, ya que abren la puerta a posibles cambios en las relaciones de pareja y suponen la superación de algunos de los roles de género tradicionales. Entre otras cuestiones, se les ha preguntado acerca de la gestión del trabajo doméstico y de cuidados, y sobre los mecanismos que utilizan en su negociación.

Como se desarrollará más adelante, hemos constatado cómo el reparto del trabajo doméstico y de cuidados ha pasado a ser, actualmente, el terreno donde se dirimen las diferencias de género en las relaciones de pareja. Al preguntar sobre la paridad en sus relaciones de pareja, la mayoría de las personas entrevistadas afirman formar parte de una pareja paritaria ${ }^{2}$ porque reparten (de manera más o menos equitativa) el trabajo de cuidados y doméstico. Son pocas las personas que contemplan o miden la paridad en otros ámbitos. Este ámbito es referenciado continuamente, al mismo tiempo que se evitan cuestiones más espinosas, como pueden ser la gestión de los recursos económicos, el espacio, otras relaciones sociales o afectivas o los intereses propios... Aún así, en las parejas con un ideal feminista más desarrollado la paridad es abordada con mayor amplitud.

1. Es una parte de una investigación llevada a cabo para una tesis doctoral dirigida por la Dra. Mari Luz Esteban Galarza con el título Procesos de negociación en modelos emergentes de parejas. Discursos y prácticas de parejas con ideología feminista, que ha contado con la financiación de las becas predoctorales del Vicerrectorado de Euskera de la Universidad del País Vasco-Euskal Herriko Unibertsitatea en la convocatoria de 2011.

2. Entendida como una relación de pareja en la que no se reproducen los roles de género tradicionales y en la cual la igualdad de género es una prioridad para sus integrantes. 
Curiosamente, aunque la negociación de estas y otras cuestiones sea fundamental para la consecución de una relación de pareja paritaria, no existe una conciencia amplia de su necesidad. La mayoría de las personas entrevistadas definen el reparto que llevan a cabo como «natural» o espontáneo, ya que la explicitación de acuerdos chocaría frontalmente con el ideal de amor romántico imperante en nuestra sociedad. Esta falta de negociación abierta y explícita dificulta el cambio de los modelos de género tradicionales, al perpetuar las desigualdades en el reparto de estos trabajos.

\section{El trabajo doméstico y de cuidados: ¿de «ayudar» a compartir?}

Cuando hablamos de trabajo doméstico y de cuidados nos referimos a todo el trabajo no remunerado que se lleva a cabo desde los hogares, dentro y fuera de estos y que, principalmente, han realizado las mujeres y, en gran medida, ha estado invisibilizado. Así se da la «paradoja del cuidado: necesario pero invisible» ${ }^{3}$, ya que se hace imprescindible para el desarrollo de la vida humana pero, por otra parte, durante mucho tiempo su estudio dentro de las ciencias sociales y económicas ha quedado relegado a un segundo plano.

Aun así, desde la década de los setenta del pasado siglo ha habido un intento de, por una parte, definir conceptualmente este trabajo y, por otra parte, visibilizar, cuantificar y revalorizarlo desde diferentes perspectivas. De esta manera, existen investigaciones monográficas, principalmente desde la sociología y la economía feminista, que cuantifican en tiempo y en dinero lo invertido en estos trabajos ${ }^{4}$, con el objetivo de visibilizar la desigualdad existente entre hombres y mujeres. Entre todos estos trabajos, cabe destacar el trabajo de María Ángeles Durán La jornada interminable ${ }^{5}$, obra pionera en el contexto del Estado español que estudia el trabajo doméstico. Esta autora plantea la contribución indispensable del trabajo doméstico para el mantenimiento de la economía capitalista y expone la necesidad de estudiar este fenómeno. De esta manera, sería posible delimitar el problema fundamental con el que nos encontramos, que es que el trabajo doméstico y de cuidados siga recayendo, fundamentalmente, en las mujeres, lo que dificulta en gran medida la consecución de una pareja paritaria.

3. CARRASCO, Cristina. «La paradoja del cuidado: necesario pero invisible». Revista de Economía Crítica, 5 (2003), pp. 39-64 (39).

4. Las más destacadas en el Estado español son: CARRASCO, Cristina. El trabajo doméstico, un análisis económico. Madrid, Ministerio de Trabajo y Seguridad Social, 1991. CARRASCO, Cristina. El trabajo doméstico y la reproducción social. Madrid, Instituto de la Mujer, 1991. Durán, Ma Ángeles. De puertas adentro. Madrid, Instituto de la Mujer, 1987.

5. DURÁN, Ma Ángeles. La jornada interminable. Barcelona, Icaria Editorial, 1986. 
Estas investigaciones concluyen que, mientras que cada vez hay más mujeres económicamente activas, la participación de los hombres en las tareas del hogar sigue siendo muy baja ${ }^{6}$. Es decir, mientras un número importante de mujeres se ha ido introduciendo en prácticas tradicionalmente realizadas por hombres, los hombres no se han ido introduciendo en la misma medida en esas otras tareas tradicionalmente realizadas por mujeres.

En esta línea se inscriben trabajos como los de Cristina Carrasco $^{7}$ y Felisa Chinchetru $^{8}$ en los que se pretende valorar el trabajo doméstico desde el contexto específico de la ciencia económica. De este modo se pretende visibilizar una parte del trabajo que se ha ocultado en la economía tradicional, para lo que se hace imprescindible, como un paso previo a cualquier análisis posterior, la medición cuantitativa de las actividades del trabajo doméstico. Cuantificar qué y cuánto suponen el conjunto de las actividades cotidianas desempeñadas en el ámbito familiar.

Por otra parte, aunque siguiendo la misma lógica, nos encontramos con un número no menos importante de investigaciones que se centran en los usos del tiempo de hombres y mujeres, con los que se pretende poner de manifiesto las desigualdades de género y la discriminación que sufren las mujeres a este respecto. Así, mediante la Encuesta de Empleo del Tiempo del Instituto Nacional de Estadística español ${ }^{9}$ queda patente la desigualdad entre hombres y mujeres en la inversión de tiempo que realizan en el ámbito familiar.

Una de las consecuencias más importantes que tiene la mayor implicación de las mujeres en el trabajo familiar, es la repercusión negativa que esto tiene en el mundo laboral, ya que la discriminación familiar de las mujeres y la discriminación laboral que éstas sufren, se retroalimenta, como lo consta$\tan$ diferentes investigaciones. Entre otras, María Ángeles Durán ${ }^{10}$, que hace una crítica política de la economía domestica, así como Cristina Borderias,

6. RoYO, Raquel. El trabajo familiar en la CAVP: una perspectiva cualitativa en parejas de doble ingreso. Tesis doctoral, 2008. Tobío, Constanza. «Cuidado e identidad de género, de las madres que trabajan a los padres que cuidan». Revista Internacional de Sociología, Bol. 70-2 (2012), pp. 399-422.

7. Carrasco, Cristina. El trabajo doméstico, un análisis económico. Op. cit.

8. Chinchertru, Felisa. Económica del trabajo de las mujeres en el caso de Euskadi. Bilbao, UPV/EHU, 1993.

9. INE-Instituto Nacional de Estadística. Encuesta de Empleo de Tiempo 2009-2010. Madrid, INE, 2011. INE-Instituto Nacional de Estadística. Encuesta de Empleo de Tiempo 20022003. Madrid, INE, 2004.

10. DURÁn, María Ángeles. La jornada interminable. Op. Cit. 
Cristina Carrasco y Carme Alemany ${ }^{11}$, que junto con otras autoras plantean las coordenadas del debate que se desarrolla actualmente entorno a la relación entre reproducción social y empleo, o el de Constanza Tobío ${ }^{12}$.

Tobío ${ }^{13}$ destaca la incompatibilidad de muchas mujeres para llevar a cabo tanto el trabajo remunerado como el trabajo familiar de manera satisfactoria, incompatibilidad que sufren en menor medida los hombres. Esta autora defiende que estas tensiones se dan, en parte, por la convivencia entre nuevos modelos laborales (entre otros, la incorporación más o menos generalizada de las mujeres al trabajo remunerado) con viejos modelos familiares (en los que la mujer sigue siendo la principal responsable del bienestar familiar y el pilar en torno al que se vertebra el trabajo de reproducción). Mientras que en el ámbito laboral se ha roto con algunos de los roles de género, si bien no de manera completamente efectiva, sí de manera «institucional», en otro espacio como es el ámbito familiar, sigue dándose una continuidad de los viejos roles de género. De esta forma, esta autora afirma que mientras que hay una conciencia muy extendida de que el empleo de las mujeres es algo necesario e, incluso, deseable, no existe, por el contrario, una conciencia de la necesidad del reparto de las tareas que antes recaían exclusivamente en las mujeres. Es decir, no existe la conciencia del trabajo masculino dentro del hogar como algo necesario y deseable. Sin embargo, son dos caras de la misma moneda, y al no incidir de igual manera en estos dos ámbitos, se crean tensiones y conflictos insalvables que tienen como consecuencia la aparición de, principalmente para las mujeres, situaciones precarias ${ }^{14} \mathrm{y}$ altamente insatisfactorias, así como graves impedimentos a la hora de prosperar laboralmente.

Pocas son las alternativas que se plantean y promueven actualmente desde la esfera política y social a la hora de conseguir una mayor racionalización en los tiempos y esfuerzos que supone el trabajo doméstico. Una de estas alternativas podría ser la simplificación del trabajo doméstico, como apunta

11. Borderias, Cristina; CARRAsco, Cristina y Alemany, Carme. Las mujeres y el trabajo: rupturas conceptuales. Barcelona, Icaria Editorial, 1994.

12. Tobío, Constanza. Madres que trabajan, dilemas y estrategias. Madrid, Cátedra Feminismos, 2005.

13. Ibíd.

14. Por ejemplo, mediante la figura de la abuela como responsable del cuidado de los y las menores o como el recurso de la ayuda doméstica externa remunerada que, en muchas ocasiones, crea situaciones todavía más precarias, siendo una fuente de empleo no regulado muy importante y dejando, a su vez, la imposibilidad de hacer compatible empleo y trabajo familiar en manos de otras mujeres de clases inferiores y, en muchos casos, inmigrantes que no cuentan con derechos sociales y laborales (Tobío, 2005: 177). 
Tobío ${ }^{15}$. Aún así, esta simplificación no se da por la alta valoración que se le imprime a todo lo «casero»y «tradicional». Una situación paradójica, ya que por una parte, hay una falta de valoración del trabajo doméstico pero, por otra parte, actualmente se está dando una revalorización de «lo hecho en casa», obviando, eso sí, el trabajo que conlleva:

En muy escasa medida se está simplificando el trabajo doméstico, seguramente por una inercia ideológica que hace que los modos más tradicionales, caseros y artesanos de hacer alcancen la mayor valoración, mientras que lo industrial y estandarizado se asocia a pérdida de calidad de vida. Con independencia de que ello responda o no a la realidad, la exigencia añadida que tal percepción supone se añade a la ya de por sí compleja doble vida de las madres que trabajan ${ }^{16}$.

Esta misma autora destaca que la cantidad de tiempo que los hombres invierten en el trabajo doméstico depende, fundamentalmente, de la edad y de la clase social aunque, en todos los casos sin excepción, sea muy inferior al tiempo que dedican las mujeres a estos trabajos ${ }^{17}$. Así, cuanto más elevado es el nivel socioeconómico de la pareja, más tareas serán repartidas o no realizadas por ningún miembro de la pareja (lo que nos lleva, de nuevo, a la creación de situaciones todavía más precarias). También la edad es una variable significativa a la hora de analizar la implicación en el trabajo familiar según esta autora que, en consonancia con otras investigaciones similares ${ }^{18}$, subraya cómo en las generaciones más jóvenes se están dando cambios hacia pautas más igualitarias. Si bien la mayoría del trabajo sigue recayendo en las mujeres y muchas de las tareas desempeñadas por los hombres tienen más un carácter complementario y no sustituto.

Cabría preguntarse por la forma que ha tomado este reparto de roles en las parejas formadas por personas del mismo sexo, y es precisamente lo que investiga Pichardo ${ }^{19}$. Este autor concluye que, en este caso, el reparto generizado de tareas no es el criterio que se sigue en la mayoría de parejas homosexuales, ya que los roles de género se han transcendido pero, en ningún caso, se han superado todo tipo de relaciones de poder, sino que suelen ser otras variables las que toman esa relevancia a la hora de definir la implicación en el reparto de tareas familiares. Aún así, aunque este reparto generizado no se dé

15. Ibíd., p. 267.

16. Ibíd.

17. Ibíd., p. 199.

18. Royo, Raquel. Op. Cit.

19. PICHARDO, José Ignacio. Entender la diversidad familiar. Relaciones homosexuales y nuevos modelos de familia. Barcelona, Edicions Bellaterra. 2009. 
dentro de estas parejas, se sigue reproduciendo de igual manera una vez que se externalizan estas tareas o cuidados. Así, en la mayoría de los casos son las abuelas las que se hacen cargo de una parte importante del trabajo, sobre todo de la crianza, pero no así los abuelos:

Es decir que, aunque simbólicamente y en la práctica se rompe de forma generalizada con la división sexual del trabajo, las personas homosexuales no escapan a la construcción de un sistema de género estructural y sistémico. De este modo, el reparto de tareas va más allá de sus opciones personales y de su relación de pareja, reproduciendo a veces la división sexual del trabajo no en el interior de la pareja, sino en contextos más amplios en los que acaban siendo mujeres las que realizan las tareas domésticas y de cuidado de los menores $^{20}$.

Esto nos da una idea de lo extendido y profundamente arraigado que está el reparto generizado del trabajo, que incluso tiende a reproducirse en contextos en los que se supone que más fácilmente debería superarse. Así, queda patente la fuerza con la que los roles de género perméan todas las esferas sociales, principalmente, mediante un determinado reparto del trabajo.

Una parte fundamental de estos trabajos tienen que ver con el cuidado, por lo que son numerosos los trabajos que han surgido en los últimos años que subrayan la «obligatoriedad de cuidar que sufren las mujeres» ${ }^{21}$. Estas autoras abordan «la crisis de cuidados» planteada al hilo del debate y aprobación de la Ley de Promoción de la autonomía personal y atención a las personas en situación de dependencia ${ }^{22}$. Al presentar las tensiones que los cuidados conllevan y que se han dado hasta el momento de manera «privada»:

La crisis de los cuidados es un problema socioeconómico de primer orden, que afecta al conjunto de la población y que solo puede percibirse en toda su magnitud si dejamos de centrar la visión en los mercados y lo monetarizado y, en cambio, situamos como categoría analítica básica la sostenibilidad de la vida ${ }^{23}$.

20. Ibíd., p. 255.

21. ESTEBAN, Mari Luz. «Género y cuidados: algunas ideas para la visibilización, el reconocimiento y la distribución», en Congreso Internacional Sare 2003: Cuidar cuesta: costes y beneficios del cuidado, Vitoria-Gasteiz, Emakunde, 2003, pp. 63-84. CARRASCO, Cristina. «La paradoja del cuidado: necesario pero invisible», Revista de Economía Crítica, 5 (2006), pp. 39-64. PÉREZ, Amaia. «Amenaza tormenta: la crisis de los cuidados y la reorganización del sistema económico», Revista de Economía Crítica, 5 (2006) pp. 7-37.

22. Aprobada en diciembre de 2006 y actualmente en claro retroceso.

23. PÉREZ, Amaia. Op. Cit. pp. 8-9. 
Así, Pérez ${ }^{24}$ considera que este es un momento adecuado para la revisión y reconfiguración de las estructuras que hacen posible que el cuidado siga recayendo siempre en las mujeres y plantea alternativas para esta situación, como es la de poner el foco de interés económico en otro sitio y no siempre en el mercado productivo.

Una vez que el trabajo de cuidados se ha problematizado, entre otras cuestiones, por la entrada de las mujeres al mercado laboral, surge el concepto de conciliación, término que ha obtenido relevancia en los últimos años y que tiene por objeto hacer compatible las diferentes esferas de la vida de una persona. Para ello, desde diferentes instituciones, se han puesto en marcha políticas públicas ${ }^{25}$.

Una de las mayores críticas que se le hace a estas políticas de conciliación es el hecho de «estar pensadas y diseñadas para mujeres» ${ }^{26}$, situando a las mujeres como el centro del problema y, por otra parte, señalando como un problema la baja tasa de empleo femenino, obviando la otra cara de la moneda, es decir, la falta de implicación masculina en el trabajo doméstico y de cuidados. Así, Torns y Moreno concluyen que:

En síntesis, puede afirmarse que las políticas de conciliación no tienen en cuenta las desigualdades de género y que, en los países con modelos de bienestar mediterráneos, las agravan. Este efecto perverso tiene su razón de ser en unas políticas que no tratan adecuadamente el tiempo de trabajo, entendido en su acepción más amplia, tal como se ha comentado. Por ello, la conciliación ofrece unas soluciones reforzadoras de la división sexual del trabajo. Unas soluciones de 'siempre` que resultan inviables para las mujeres jóvenes de hoy. Bien porque no revisan el reparto de la carga total de trabajo y, en especial, del trabajo doméstico y de cuidado, bien porque se destinan, exclusivamente, a las madres activas laboralmente. Una estrategia que, a su vez, poco coincide con las tendencias demográficas actuales, caracterizadas por la baja fecundidad ${ }^{27}$.

Como comentan estas autoras, una de las políticas de conciliación son las excedencias para el cuidado de menores. Si nos detenemos en el número de excedencias que se solicitan para este fin podemos observar la gran diferencia que existe dependiendo del sexo de la persona que lo solicita. Durante los

24. Ibíd.

25. BORRÁs, Vicent; TORNS, Teresa; MORENO, Sara: «Las políticas de conciliación: políticas de empleo versus políticas de tiempo». Papers 83 (2007), pp. 83-96.

26. TORNS, María Teresa y MORENO, Sara: «La conciliación de las jóvenes trabajadoras: nuevos discursos, viejos problemas», Revista de Estudios de Juventud 83 (2008), pp. 101-117 (102).

27. Ibíd.

Feminismo/s 23, junio 2014, pp. 91-110 
años 2008, 2009 y 2010 en la CAV, alrededor de un 97\% de las excedencias fue solicitado por mujeres, sin sufrir apenas variación durante los tres años ${ }^{28}$. Mientras que las reducciones de jornada para el cuidado de menores para esos mismo años fueron solicitadas por mujeres en el 95\% de los casos. Estos datos nos presentan claramente que el cuidado de las niñas y niños, por lo menos, durante los primeros años de vida, sigue recayendo en las mujeres, a pesar de algunos intentos fallidos por fomentar la participación activa de los hombres ${ }^{29}$.

Este reparto del cuidado se establece mediante diferentes mecanismos; uno de los puntos de partida es el reparto diferenciado de las prestaciones por maternidad y paternidad, que sigue en la misma línea que las reducciones de jornada laboral y las excedencias. Esta prestación es de 16 semanas, de las que 6 semanas son de descanso obligatorio para la madre y las 10 restantes pueden ser compartidas o «utilizadas» únicamente por el otro progenitor. En la CAV el porcentaje de hombres que accede a esta prestación es «una rareza estadística» que no supera el $5 \%$ en ningún caso ${ }^{30}$.

Estos datos nos hacen suponer que el reparto de las tareas que se llevan a cabo en la mayoría de los hogares en el momento de la crianza, es un reparto desigual basado en las diferencias de género. Aunque las bajas de maternidad sólo hacen referencia a las primeras 16 semanas de las criaturas, este primer reparto desigual supondrá un precedente que se volverá permanente, lo que se comprueba con el porcentaje desigual en las excedencias y reducciones de jornada.

Por otra parte, como destaca Esteban ${ }^{31}$, la obligatoriedad de cuidar a la que se ven sometidas las mujeres, sigue siendo uno de los escollos principales a la hora de avanzar en la igualdad en el reparto del trabajo de cuidados:

28. EMAKUNDE. Cifras sobre la situación de mujeres y hombres en Euskadi 2011. VitoriaGasteiz, Emakunde, 2011.

29. «La Comunidad Autónoma Vasca decidió aumentar a los hombres la cantidad que pagaba por excedencia (estableciendo una diferencia de 600 euros respecto a lo que cobraban las mujeres). Además de discriminar a las mujeres con un pago inferior, esta medida no consiguió aumentar el número de hombres que se tomaban excedencias: 372 hombres frente a las 12.246 mujeres en 2009 (de hecho, por esa razón se eliminó esta diferencia en el importe de las excedencias en 2010, estableciendo la equiparación de las mismas así como su mejora económica hasta la cuantía de 3.500 euros). Si se hubieran analizado con detenimiento las experiencias de otros países, se hubiera sabido de antemano que ninguna de estas campañas $e$ incentivos iban a funcionar: como veremos en este libro, los hombres no se toman ningún permiso que puedan ceder a las mujeres o que no reemplace sustancialmente sus ingresos del trabajo dejados de percibir». (Castro y Pazos, 2011: 7).

30. EMAKUnde, Op. Cit.

31. ESTEBAN, Mari Luz, «Género y cuidados: algunas ideas para la visibilización, el reconocimiento y la distribución» Op. Cit. pp. 4-5. 
Como concuclusión general podemos decir que las medidas que se están tomando no están suponiendo la reversión de la situación, en cuanto que la responsabilidad de los cuidados sigue estando en manos de las mujeres, y no se está cuestionando la «obligatoriedad» para ellas, lo que debería ser una condición sine qua non ${ }^{32}$.

Por otra parte, esta misma autora destaca que las bases de la ideología de la maternidad imperante en la actualidad en occidente ahondan en este reparto desigual $^{33}$. Esta ideología maternal destaca dos cuestiones fundamentales: la importancia del cuidado de las criaturas por parte de la madre, sobre todo en los dos primeros años, y la promoción de la lactancia materna. Todo esto neutraliza, en cierta medida, los esfuerzos por la corresponsabilidad del trabajo doméstico y la crianza, y la compatibilidad entre éste trabajo y el empleo.

Es por todo esto, por lo que diferentes autoras ponen en entredicho que la finalidad de muchas políticas sociales diseñadas actualmente sea la de compatibilizar el empleo con la crianza. Por una parte, como ya hemos mencionado, las mujeres parecen ser las únicas destinatarias de estas políticas y, por otra, en vez de compatibilizar, lo que parecen buscar es una alternativa a la escasa red de servicios públicos (tales como guarderías, servicios de cuidados de día...). Esto supone «retirar» a las mujeres madres del mercado laboral, si no para siempre, sí por un tiempo determinado, con todos los problemas que esto conlleva en cuanto a la reincorporación al puesto de trabajo y dependencia económica:

Las políticas sociales no siempre tienen como objetivo la compatibilidad entre las responsabilidades familiares y laborales. En algunos casos, pretenden más bien retirar temporalmente de la actividad laboral a las madres para que se puedan dedicar al cuidado de los hijos(...). Se trata de dispositivos que tienden a producir una selección según características socioeconómicas y de cualificación: son las mujeres con menos oportunidades laborales por su menor formación las que en mayor medida deciden disfrutar de estas ayudas, lo cual produce la tendencia a la diferenciación entre cuidadoras y mujeres liberadas de estas tareas ${ }^{34}$.

Ante este panorama de desigualdad nos encontramos con diferentes situaciones y estrategias de reparto en nuestro estudio empírico, como veremos a

32. Ibíd.

33. ESTEBAN, Mari Luz: «La maternidad como cultura: algunas cuestiones sobre lactancia materna y cuidado infantil», en Enrique Perdiguero y Josep M. Comelles (eds.): Medicina y cultura: estudios entre la antropología y la medicina, Barcelona, Edicions Bellaterra, 2000, pp. 207-226.

34. Toвío, Constanza. Madres que trabajan, dilemas y estrategias. Op. Cit. p. 270.

Feminismo/s 23, junio 2014, pp. 91-110 
continuación. Estudio que se basó en la realización de entrevistas en profundidad a 15 parejas del País Vasco que consideraban que tenían una relación paritaria.

\section{Las negociaciones en torno a los trabajos domésticos y de cuidados}

Como hemos señalado con anterioridad, las personas entrevistadas se autodenominaban paritarias y definían su relación como igualitaria. Una de las cuestiones principales sobre las que se les preguntó fue por qué se consideraban así y dónde creían que residía la paridad. En la mayoría de los casos el concepto de paridad y el reparto de los trabajos domésticos y de cuidados iban de la mano, ya que el primer aspecto al que hacían referencia era este: «Sí, sí, sí, incluso a veces él hace más cosas que yo. Bueno, ¿qué quieres decir, en cosas de casa y así...?»(10.1. Mujer, 28, relación heterosexual) ${ }^{35}$. Pocas han sido las personas que hacen referencia a otros aspectos de la relación de pareja, a pesar de pedirles que profundizaran en lo que supone ser una pareja paritaria. Esto puede ser debido a que el reparto de estos trabajos es un tema que ha adquirido relevancia social, se ha creado y extendido un discurso al respecto que otorga centralidad a estas prácticas a la hora de medir las diferencias de género.

Es por ello que podríamos afirmar que este tema ha sido una constante durante toda nuestra investigación, al preguntarles sobre otras cuestiones como son las negociaciones, los acuerdos, las discrepancias etc., esta era la cuestión que primero afloraba. Por lo tanto, es un tema capital en la configuración de las relaciones de pareja que nos muestra, no solo cómo se configura ese reparto, sino que nos deja entreveer sobre qué relaciones de poder se asientan las relaciones de pareja. De alguna manera, son el térmometro que mide el funcionamiento de la pareja y, más concretamente, el nivel de paridad en el que viven. Igualmente, es un espacio privilegiado que nos permite ver cómo se llevan a cabo las negociaciones, acuerdos y desacuerdos cotidianos, ya que se ha convertido en el campo de batalla de muchas de las reivindicaciones que se hacen en el seno de las parejas. Es por ello por lo que acercándonos a esta realidad podemos conocer también otras dinámicas y procesos negociadores. Así lo destaca Esteban ${ }^{36}$, ya que considera que son una cuestión fundamental y que las tensiones que aparecen en la relaciones de pareja acerca

35. La información que aparece entre paréntesis es la siguiente: número de identificación de la persona entrevistada, sexo, edad y tipo de relación que mantenían en el momento de realizarse la entrevista.

36. Esteban, Mari Luz. Crítica del pensamiento amoroso. Barcelona, Edicions Bellaterra, 2011. p. 384 
de este tema, son el reflejo de las tensiones entre mujeres y hombres; una metáfora de la igualdad no conseguida y de los obstáculos aún por superar.

Aunque son innegables los cambios acaecidos en las sociedades occidentales en lo que se refiere a igualdad formal y a la tasa de empleo de las mujeres, estos cambios no se corresponden, en la misma medida, con cambios en el ámbito considerado como privado. Como destaca Tobío ${ }^{37}$, las mayores tasas de empleo femenino no viene parejas a una mayor dedicación de los hombres en los trabajos domésticos y de cuidados.

Prácticamente todas las personas entrevistadas tenían un empleo, tanto mujeres como hombres, pero la implicación en los trabajos domésticos y de cuidados era mayor en las mujeres que en los hombres. Por ello, si bien es cierto que el tema del trabajo doméstico y de cuidados ha sido una constante a lo largo de la investigación, ha sido entre las mujeres entrevistadas donde se ha hablado más y más detalladamente del mismo, no así entre ellos.

Aunque entre las personas entrevistadas se ha visto un reparto de roles generizado, no podemos asegurar que se de un único modelo, ya que las diferencias son significativas. Por otra parte, el intento por superar el reparto sexual de los roles se ha visto en la mayoría de las parejas, principalmente, por parte de las mujeres, que tienen una actitud crítica al respecto e intentan redefinir los roles y los trabajos adscritos a los mismos. Así, el detonante para los cambios suelen ser las reivindicaciones de las mujeres. Podemos afirmar, por lo tanto, que las mujeres son las que muestran mayor interés en un cambio de modelo, porque son las que más sufren situaciones de desigualdad y los hombres, por el contrario, suelen estar satisfechos con su situación, si bien es cierto que a nivel simbólico también plantean discrepancias con el modelo de pareja tradicional.

En cuanto a las parejas homosexuales, puede decirse que se aprecian diferencias, puesto que el reparto de estos trabajos no se realiza en base a un reparto generizado, sino que son otras las variables en torno a las que se establece, como son la edad y la situación laboral. Por lo tanto, en estas parejas este trabajo no es tanto un termómetro de igualdad de género como lo es en las parejas heterosexuales.

En general, el reparto de roles en las parejas entrevistadas se ha venido estableciendo a lo largo del tiempo de la relación y tiende a mantenerse inmutable, ya que lo que se suponía que eran acuerdos puntuales, se solidifica, lo

37. Toвío, Constanza. «Cuidado e identidad de género, de las madres que trabajan a los padres que cuidan». Op. Cit.

Feminismo/s 23, junio 2014, pp. 91-110 
que dificulta su redefinición. Algunas de las entrevistadas son conscientes de ello:

Pero, yo creo que en una pareja es un poco a ver quién se sube al carro primero. Si tú empiezas haciendo cositas... ya el otro se acostumbra... y para el otro es perfecto, llegar de trabajar y no tener que hacer nada, entonces ahí se establece como unas cosas, hay como unos roles... que ya... cuando tú intentas cambiarlo suele ser tarde (4.1. Mujer, 46, relación heterosexual).

Aunque la mayoría de las personas entrevistadas creen que un reparto equitativo de estos trabajos es fundamental para el bienestar de la pareja, este reparto no suele concretarse. Así, en la mayoría de parejas el reparto de este trabajo no suele explicitarse, como manera de evitar los conflictos pero esto, a su vez, conlleva que se mantengan los roles tradicionales. Es decir, la falta de explicitación y negociación en el reparto de los trabajos domésticos y de cuidados perpetúa un reparto desigual basado en los roles tradicionales de género. Así, se tiene la percepción de que es el devenir «natural» de la relación de pareja el que posibilita un reparto u otro: «También ha sido muy natural, no ha habido que hacer una repartición de las tareas» (3.2. Hombre, 31, relación heterosexual). Por lo tanto, nos encontramos con una situación algo contradictoria: existe la conciencia de la necesidad de reparto pero no se ve necesario acordarlo:

Sale de manera natural, y vas viendo... no hacemos una tabla de ningún tipo, es la casa de los dos, el espacio de los dos, los dos tenemos que trabajar y no estamos contando: yo hoy he lavado tres platos, pues no, lo que surge, ahi está, pues lo limpias y ya (8.1. Hombre, 31, relación heterosexual).

Así lo cuenta otro de los entrevistados: «Luego van saliendo las cosas, no hay tareas específicas para cada uno ni nada por el estilo, ni un organigrama, si van saliendo las cosas, pues se hacen...» (7.1. Hombre, 35, relación heterosexual).

A pesar de que se sienta que hay un «reparto natural» de estos trabajos, las mujeres suelen mostrar su disconformidad con esta situación y son las que suelen plantear reivincidaciones de cambio, ya que saben que existen alternativas a ese reparto supuestamente natural: «Hago yo más y yo creo que se da por hecho que yo hago más por ser mujer, pero también pasa que si no hay hecho algo que se supone que tengo que hacer yo, nadie dice nada» (6.2. Mujer, 28 , relación heterosexual).

En esta cita anterior podemos comprobar cómo espacios que se suponen que ocupaban las mujeres han ido vaciándose, como plantea Tobío ${ }^{38}$, pero

38. ToBío, Constantza. «Cuidado e identidad de género, de las madres que trabajan a los padres que cuidan». Op. Cit. 
mientras que las mujeres han dejado espacios vacios para ocupar espacios tradicionalmente masculinos, esos espacios no se han visto ocupados por hombres. Así, se ha dado un «vaciamiento» de los espacios domésticos y esto conlleva conlictos y problemas logísticos:

Hombre eso sí me cabrea mucho, que nunca se acuerda, que nunca se acuerde de... ni nunca piensa en qué va a necesitar si nos vamos de aquí. Entonces soy yo siempre la que tiene que llevar los pañales, el no sé qué, el tal... la comida, la merienda... Y cuando estamos por ahí: ¿hemos traído...? ¿Hemos traído...? ¿Cómo que hemos traído...?, ¡No! Ese es el único tema que me repatea un poquito (7.2. Mujer, 31, relación heterosexual).

Aún así, algo a lo que se suele hacer referencia en la mayoría de las entrevistas es a que las cosas están cambiando, sobre todo, entre las personas más jóvenes. Se tiene la sensación de que situaciones que en generaciones anteriores eran la norma, hoy en día son excepción. Al igual que los trabajos domésticos y de cuidados se utilizan como termómetro de la igualdad, también se utiliza la comparación con otras parejas.

Aunque, como hemos señalado, la mayoría de los testimonios recogidos hacen referencia al reparto de tareas como natural, hay unas pocas personas, principalmente mujeres, que creen que explicitar ese reparto es importante para que sea un reparto adecuado. Así, se llevan a cabo diferentes estrategias que ponen en evidencia el reparto desigual, como medida para llegar a una situación más justa:

A veces hago cosas para poner en evidencia que yo hago más y que no estoy dispuesta a que se quede como algo establecido, por lo menos, que quede patente que esto es así y que no se dé por sentado que lo tengo que hacer yo. En esas situaciones, sobre todo, es cuando me doy cuenta y yo creo que él también se da cuenta que yo hago algo más (6.2. Mujer, 28, relación heterosexual).

Aún siendo consciente de la necesidad de verbalizar este reparto, se encuentran resistencias y obstáculos, así lo cuenta esta mujer, militante feminista que hace alusión a la utilización de un instrumento que mida ese reparto de tareas, como es el «berdinometro ${ }^{39}$ :

No hacemos nada... a pesar de que yo le amenacé con el berdinometro, pero no hemos llegado hasta ahí, esto para él es un gran conflicto. Si lo escribes,

39. Se trata de una herramienta preparada por Emakunde: «Se trata de un "juego" que permite de una forma divertida comprobar y hacer visible cómo es el reparto de las tareas domésticas en la pareja y facilitar, en su caso, un reparto más equilibrado de éstas». <http:// www.berdingune.euskadi.net/u89-congizon/es/contenidos/enlace/enlaces_mochila_ gizonduz/es_gizonduz/adjuntos/berdinometro.jpg> consultado el 16-01-2014. 
en mi opinión, se ve mucho mejor, qué cosas se hacen... qué se olvida... Porque las cosas que tú haces, no se quedan registradas, pero esto ya le parece... ponerse a malas, no sé cómo decirlo. Y al final no lo hemos utilizado (12.1. Mujer, 34, relación heterosexual).

Al reivindicar una situación más justa, hay hombres que se sienten atacados, como podemos ver en el comentario anterior. De esta manera, la mujer no lleva a cabo lo que tienen pensado, a pesar de parecerle lo más adecuado y justo, porque le parece «violento». De esta manera, parece ser que explicitar negociaciones y acuerdos sobre el reparto de estos trabajos choca directamente con el ideal de amor romántico imperante en nuestra sociedad. El amor romántico que todo lo puede y no debe ser mezclado con cuestiones mundanas como el trabajo doméstico y de cuidados.

De la misma manera, a la hora de negociar sobre este reparto, existe el sentimiento entre las mujeres entrevistadas de que ellas son sus propias enemigas: "Y yo lo quiero hacer las cosas a mi manera, y como a él le da igual no hacerlo a su manera, pero como a mí no me gusta su manera de hacer las cosas, pues al final acabo haciéndolo yo» (13.1. Mujer, 32, relación heterosexual).

Así lo destaca esta otra entrevistada:

Por ejemplo, a mí no me gusta ver el fregadero lleno de cacharros, y como a él no le importa, entonces, claro, si yo lo friego ¿qué es, que yo he hecho más trabajo? Sí, pero no tengo por qué hacerlo. Y claro, las cosas se lían mucho ahí, quién hace más... quién hace menos... pues vamos a decidir. Igual haces más porque sientes más esa necesidad, ahora bien, por qué sientes esa necesidad eso ya no lo sé (13.1. Mujer, 32, relación heterosexual).

Esta misma opinión se recoge en otra entrevista en referencia a los trabajos de cuidados:

A algunos, por que no se les deja, porque no es solo su culpa, las madres no dejan, las madres tienen a los niños súper protegidos y encima tienen un poder sobre ellos que son las que cortan el bacalao y que no se meta nadie, ni el marido. Si el niño está enfermo, yo soy la que sé qué tiene que tomar, cada cuanto... me voy con las amigas al cine, pero he dejado todo preparado y escrito, qué es lo que tiene que hacer el otro. Y al fin y al cabo, a mí me parece que eso es marcar quién tiene el poder, marcar el territorio, y el otro ahí sin responsabilidades. Si tu le dejas todo preparado, el otro lo hará, pero mandado por ti, y eso, por ejemplo, en nuestra pareja no es tan evidente (15.1. Mujer, 46, relación homosexual).

Concretamente, se está refiriendo a que en su pareja de dos mujeres los roles de género no están tan marcados, en general, y en el ejercicio de la crianza, en particular. 
Como ya hemos mencionado, este tipo de opinión recogida en la cita anterior, se repite en diferentes entrevistas en las que queda patente la falta de implicación de los hombres, tanto en el trabajo doméstico como en el de cuidados, pero se le otorga diferentes justificaciones.

A este respecto, Tobío $^{40}$ sintetiza la falta de implicación de los hombres en la crianza en tres aspectos: porque no saben, porque no pueden y porque no quieren. Estos tres motivos estarían interrelacionados. No se les educa para cuidar, por lo que no lo ven necesario y, por lo tanto, no quieren hacerlo; y no pueden hacerlo, porque su dedicación laboral no se configura desde la necesidad de los cuidados, como sería en el caso de las mujeres, sino que su disponibilidad para cuidar depende de su dedicación laboral. Además, a ejercer la crianza, tradicionalmente, se les ha enseñado a las mujeres, los hombres no han participado de ello. Por último, no quieren cuidar, porque no sienten que sea su responsabilidad e invierten el tiempo en otras actividades que adquieren una mayor valoración social.

El inicio de la crianza es un momento especialmente significativo en el reparto de roles y de carga de trabajo, ya que es un momento de redefinición que configurará, en gran medida, el modelo a seguir. De hecho, es un momento propicio en el que las desigualdades de género tienden a agrandarse $^{41}$. Esto tiene que ver con un modelo de maternidad que cobra fuerza, como es la maternidad intensiva, que otorga centralidad a la figura materna por encima de la paterna ${ }^{42}$. Junto con esto, como ya hemos apuntado anteriormente, el desigual disfrute de los permisos de maternidad y paternidad, configuran un reparto del trabajo de cuidados desigual, en el que son las mujeres las que tienen que asumir la mayoría de las renuncias: «Pues yo veo a mi alrededor que los hombres no dejan sus hobbies, o sea, el que jugaba a futbito, sigue jugango, pero las mujeres sí, sí que suelen dejar de hacer lo que hacían» (15.1. Mujer, 46, relación homosexual).

De esta manera, muchas mujeres feministas, se encuentran en la encrucijada de practicar la maternidad intensiva pero con miedo de perder parte de su identidad y de las relaciones sociales que ello conlleva:

40. Ibíd.

41. Castro, Carmen y Pazos, María: «Hombres, cuidados e igualdad de género. Fundamentos para la equiparación efectiva entre los permisos de padres y madres», comunicación presentada en el Congreso Iberoamericano de masculinidades y equidad: investigación y activismo, Barcelona, <http://www.redmasculinidades.com/sites/default/files/ archivos/biblioteca/00246.pdf> consultado 08-01-2014.

42. HAYs, Sharon. Las contradicciones culturales de la Maternidad. Barcelona, Paidós, 1998.

Feminismo/s 23, junio 2014, pp. 91-110 
De la última relación he aprendido lo que no quiero, él no se daba cuenta pero yo estaba anulada como persona, yo era madre pero solo madre, nada más. Entonces 8 años estuve con ellos en casa todo el día y nada más. No salía, no estaba con amigas y estaba muy atada, a las ocho a casa, los niños a la cama y él, todo inconsciente, decía, bueno, yo ya he hecho, ahora me voy a una reunión aquí, a no sé dónde... y yo no iba a ningún lado. Alguna vez si decía que iba a salir: jo, ¿para qué? ¡si hace frío!. O sea, no me decía que no, pero me cortaba el rollo, no me ayudaba, yo necesitaba que me empujaran un poco... y al final tanto tiempo así, pues te cuesta y al final te da miedo salir a la noche...(14.2. Mujer, 30, relación homosexual).

Por lo tanto, nos encontramos con una situación que supone una encrucijada para muchas mujeres, entre lo que tradicionalmente se espera de ellas y la concepción que tienen de la paridad, lo que conlleva profundas distorsiones, tensiones y conflictos.

\section{A modo de conclusión}

Es fundamental negociar el trabajo doméstico y de cuidados para la consecución de una pareja igualitaria. Si bien es cierto que se utiliza este reparto como termómetro a la hora de medir la paridad de una pareja, existe la convicción de que el reparto surge de manera «natural». Al no explicitarse este reparto «natural» se dificulta su reorganización, lo que redunda en un reparto desigual basado en los estereotipos de género. Por lo tanto, la falta de explicitación conlleva la perpetuación del reparto desigual. Así, el amor romántico neutraliza la utilización de estrategias negociadoras que se utilizan en otros ámbitos (laborales, relaciones de amistad, otras relaciones familiares...). Por todo ello, muchas de las personas entrevistadas parecen percibir como incompatibles la negociación abierta y sin ambages y el ideal de amor romántico. Es decir, la manera de entender los lazos afectivos diluye el carácter negociador de algunas decisiones en la relación de pareja.

De la misma manera, no se explicitan todos los posibles conflictos o diferencias de criterio dentro de la pareja, ya que negociar estas diferencias supone aceptarlas, lo que entra en contradicción con el ideal de amor imperante, que tiende a identificar amor romántico con afinidad total y a disparidad de criterios con incompatibilidad. Por lo que en algunas ocasiones se confunde lazos afectivos con «entrega total»y se convierte a los procesos negociadores en algo problemático. Esto conlleva la perpetuación o la continuidad de algunos modelos relacionales, tales como el reparto generizado de las tareas y obligaciones, así como una situación de subordinación de un miembro de la pareja respecto al otro. De esta manera, la explicitación de pactos que afectan a la 
relación de pareja, contribuye en el surgimiento y mantenimiento de modelos de relaciones más igualitarias.

En las parejas paritarias se favorece el reparto de trabajos domésticos y de cuidados y se plantea esto como una cuestión fundamental a la hora de definir la relación. Sin embargo, existe una distorsión entre la percepción y la realidad. Aunque en el plano ideológico se defienda el reparto equitativo, esto no se traduce en prácticas paritarias reales. A pesar de que existen cambios entre las parejas más jóvenes, ese mismo discurso paritario enmascara en ocasiones comportamientos desiguales. Es decir, la mera declaración de intenciones, mediante el discurso paritario, neutraliza la necesidad de cambiar comportamientos concretos.

Por todo esto, el reparto de estos trabajos entre las parejas heterosexuales sigue recayendo, principalmente, en las mujeres. En las parejas homosexuales, también se da una especialización que conlleva que un miembro de la pareja se ocupe más de las tareas domésticas y de cuidados que el otro miembro, pero suele estar basado en otras variables como son la edad o la situación laboral.

\section{Referencias bibliográficas}

Borderias, Cristina; CARRASCO, Cristina y Alemany, Carme. Las mujeres y el trabajo: rupturas conceptuales. Barcelona, Icaria Editorial, 1994.

BORRÁS, Vicent; TORNS, Teresa; MORENO, Sara: «Las políticas de conciliación: políticas de empleo versus políticas de tiempo». Papers 83 (2007), pp. 83-96.

CARRASCO, Cristina. El trabajo doméstico, un análisis económico. Madrid, Ministerio de Trabajo y Seguridad Social, 1991.

- El trabajo doméstico y la reproducción social. Madrid, Instituto de la Mujer, 1991.

- «La paradoja del cuidado: necesario pero invisible». Revista de Economía Crítica, 5 (2003), pp. 39-64.

- «La paradoja del cuidado: necesario pero invisible», Revista de Economía Crítica, 5 (2006), pp. 39-64.

CASTRO, Carmen y PAZOS, María: «Hombres, cuidados e igualdad de género. Fundamentos para la equiparación efectiva entre los permisos de padres y madres», comunicación presentada en el Congreso Iberoamericano de masculinidades y equidad: investigación y activismo, Barcelona, <http://www.red masculinidades.com/sites/default/files/archivos/biblioteca/00246.pdf> consultado 08-01-2014.

Chinchertru, Felisa. Económica del trabajo de las mujeres en el caso de Euskadi. Bilbao, UPV/EHU, 1993.

DURÁN, Mã Ángeles. La jornada interminable. Barcelona, Icaria Editorial, 1986. 
- De puertas adentro. Madrid, Instituto de la Mujer, 1987.

EMAKUNDE. Cifras sobre la situación de mujeres y hombres en Euskadi 2011. VitoriaGasteiz, Emakunde, 2011.

- Berdinometro. <http://www.berdingune.euskadi.net/u89-congizon/es/contenidos/enlace/enlaces_mochila_gizonduz/es_gizonduz/adjuntos/berdinometro.jpg> consultado el 16-01-2014.

ESTEBAN, Mari Luz: «La maternidad como cultura: algunas cuestiones sobre lactancia materna y cuidado infantil», en Enrique Perdiguero y Josep M. Comelles (eds.): Medicina y cultura: estudios entre la antropología y la medicina, Barcelona, Edicions Bellaterra, 2000, pp. 207-226.

- «Género y cuidados: algunas ideas para la visibilización, el reconocimiento y la distribución», en Congreso Internacional Sare 2003: Cuidar cuesta: costes y beneficios del cuidado, Vitoria-Gasteiz, Emakunde, 2003, pp. 63-84.

- Crítica del pensamiento amoroso. Barcelona, Edicions Bellaterra, 2011. p. 384.

HAYS, Sharon. Las contradicciones culturales de la Maternidad. Barcelona, Paidós, 1998.

INE-Instituto Nacional de Estadística. Encuesta de Empleo de Tiempo 2009-2010. Madrid, INE, 2011. INE-Instituto Nacional de Estadística. Encuesta de Empleo de Tiempo 2002-2003. Madrid, INE, 2004.

PÉREZ, Amaia. «Amenaza tormenta: la crisis de los cuidados y la reorganización del sistema económico», Revista de Economía Crítica, 5 (2006) pp. 7-37.

PICHARDO, José Ignacio. Entender la diversidad familiar. Relaciones homosexuales y nuevos modelos de familia. Barcelona, Edicions Bellaterra. 2009.

ROYO, Raquel. El trabajo familiar en la CAVP: una perspectiva cualitativa en parejas de doble ingreso. Tesis doctoral, 2008. TOBío, Constanza. «Cuidado e identidad de género, de las madres que trabajan a los padres que cuidan». Revista Internacional de Sociología, Bol. 70-2 (2012), pp. 399-422.

ToBío, Constanza. Madres que trabajan, dilemas y estrategias. Madrid, Cátedra Feminismos, 2005.

TORNS, María Teresa y MORENO, Sara: «La conciliación de las jóvenes trabajadoras: nuevos discursos, viejos problemas», Revista de Estudios de Juventud 83 (2008), pp. 101-117. 